\title{
Quinto Curcio ante sus fuentes: el episodio de Alejandro y los escitas del Tanais
}

\author{
Luis BALlesteros PAstor \\ Universidad de Sevilla \\ lbpastor@us.es
}

Recibido: 5 de febrero de 2015

Aceptado: 16 de marzo de 2015

\section{RESUMEN}

Quinto Curcio encontró en sus fuentes un discurso en el que un escita censuraba a Alejandro, seguido de la réplica del rey. Curcio resumió drásticamente este segundo discurso para dar realce a la crítica al macedonio. Las palabras del escita tienen un sorprendente lenguaje retórico y ciertas alusiones tomadas de la literatura griega, además de posibles referencias indirectas a Calígula. Curcio dice seguir fielmente a su fuente para justificar estas incongruencias, pero también para solapar las manipulaciones que él ha realizado con el fin de conseguir sus propios fines narrativos.

Palabras clave: Quinto Curcio. Escitas. Alejandro. Calígula. Historiografía.

\section{Quintus Curtius before his Sources: the Episode of Alexander and the Scythians on the Tanais}

\begin{abstract}
Quintus Curtius found in his sources a speech where a Scythian censured Alexander, followed by the King's reply. Curtius drastically abridged this second discourse in order to highlight the criticism of the Macedonian. The Scythian's words have a striking rhetorical language and some allusions taken from Greek literature, in addition to possible indirect references to Caligula. Curtius declares that he follows his source word-for-word aiming to justify these inconsistencies, but also trying to hide the manipulations he has done to achieve his own narrative purposes.
\end{abstract}

Key words: Quintus Curtius. Scythians. Alexander. Caligula. Claudius. Historiography.

Sumario: 1. Introducción. 2. La embajada escita: una escena tópica. 3. Situaciones inverosímiles: ¿un episodio interpolado en el relato? 4. Un escita demasiado instruido. Reflexiones sobre la fuente de Curcio. 5. Unos bárbaros no tan virtuosos: la elección de Curcio ante discursos pareados. 6. ¿Alusiones indirectas a Calígula en el discurso escita? 7. El dilema de Alejandro y las fronteras del imperio romano. 8. Conclusión. 
A la memoria de Brian Bosworth

\section{Introducción}

Conocer de qué manera abordaban los historiadores antiguos el tratamiento de sus fuentes es uno de los retos más trascendentales para el investigador moderno. Resulta decisivo saber si los textos que utilizaban aquellos autores para sus relatos eran meramente copiados, fundidos con otros o modificados con una intención premeditada. De este modo se puede indagar sobre las características de las obras que estudiemos, sobre su veracidad y, con ello, sobre su aportación para el estudio de la Historia. ${ }^{1}$

Mucho se ha escrito sobre las fuentes que empleó Quinto Curcio para su biografía de Alejandro Magno, y también sobre la manipulación que el autor latino realizó sobre las mismas. ${ }^{2}$ En el presente trabajo nos centraremos en el episodio que describe el encuentro del rey macedonio con los escitas del Tanais. En el año 329 a.C. Alejandro llega a las orillas de este río, que en realidad era el Iaxartes (Sir-Daria), pero que el rey confunde con el actual Don, creyendo por tanto que se halla en lo que los antiguos consideraban límite entre Asia y Europa. ${ }^{3}$ Según Curcio, Alejandro recibe allí una embajada de escitas que le recriminan por su ambición y le advierten de los peligros de luchar contra ellos. ${ }^{4}$ El macedonio, desoyendo estas admoniciones, consigue cruzar el río y obtiene un brillante triunfo sobre este pueblo, al que algunos consideraban invicto desde que Ciro el Grande muriera a manos de los maságetas y Darío I sufriera una severa derrota frente a los escitas europeos. ${ }^{5}$

La significación de este episodio narrado por Curcio tiene diversas facetas, todas ellas de gran interés. Desde el punto de vista de la empresa de Alejandro, alcanzar de nuevo Europa representaba completar un ciclo, demostrando el dominio macedonio

1 Para discusión general sobre este tema, véase BoswORTH 2003.

2 Para un repaso de las fuentes de Curcio, véase ante todo BARdon 1947; AtKinson 1980, 48-67; ID. 1998, 3458-3465; BAYNHAM 1998, 57-85; YARDLEY - ATKINSON 2009, 19-32.

3 Esta confusión ya fue detectada por los antiguos, y era debida a la imagen que se tenía de la forma de la Tierra: Plu., Alex., 45.5; Str. 11.7.4; Plin., NH, 6.49; Solin. 49.5; HAmilton 1971; Rickly 1975, 30; BosworTH 1980, 377-378, ID. 1995, 15, 22. También el paisaje en ambas regiones era similar, a lo que se unía el hecho de estar habitadas por escitas: Bosworth 1995, 27; Geus 2003, 238. Ya Aristóteles, Mete. 1.13 (350a), había confundido el Iaxartes con el Araxes, al que consideraba fuente del Tanais: PronTERA 1988, 87-88; Y ARDLEY HeCKel 1997, 219, y para un estudio detallado, véase RAPIN 2014.

4 Esta embajada aparece únicamente en Curcio (7.8.12-30), y ha sido considerada como uno de los puntos culminantes en su relato: Helmreich 1927, 212; BARdon 1947, 126; ANDrÉ 1990, 21; Baynham 1998, 86-89; Atkinson 2000, 45-46; Ballesteros Pastor 2003; ID. 2011; Bosworth 2004, 557-558.

5 Sobre este combate de Alejandro véase sobre todo Curt. 7.9.2-19 y Arr., An., 6.4.4-5.1; para otras referencias, Arr., An., 7.10.7; Plu., Alex., 45.6; Mor. 341c; Ep.Metz. 8; cf. D.S. 1.55.4. Arriano no menciona el nombre del río en el primero de estos pasajes, pero sí en el segundo, perteneciente al discurso de Alejandro en Opis. La ausencia de alusiones a este episodio en el Epítome de Justino puede deberse al interés de este autor por la gloria de los escitas: véase BALLESTERos PASTOR 2013, 6-10 y passim. Para el contexto histórico del episodio, véase HAMmond 1992, 274-275; Bosworth 1995, 22-33; ID. 1996, 149-150. Sobre la muerte de Ciro véase ASHERI - LLOYD - CoRCELla 2007, vol. I, 216; sobre la derrota de Darío, Ibíd. vol. II, 561-568, 642-669. Alejandro tiene presente esta debacle: Arr., An., 4.4.3. Sobre la imbatibilidad de los escitas, véase en general Ballesteros Pastor 2013, y para estos episodios, 139-141, 144-5, 286. 
sobre las dos partes del mundo y evocando en cierto modo el cruce del Danubio por las tropas de Darío, tiempo atrás. ${ }^{6}$ Desde el punto de vista estratégico, Alejandro muestra una gran inventiva para hacer pasar a su ejército por un río caudaloso y derrotar al enemigo que está en la orilla opuesta. ${ }^{7}$ El triunfo sobre los escitas confirma a Alejandro como rey invicto, ante quien ningún pueblo puede enfrentarse. Junto a ello, el macedonio consigue llegar hasta lo que él consideró confines del viaje de Dioniso hasta Oriente. De nuevo el gran conquistador emula a este dios, tan relevante en su concepción de la realeza. ${ }^{8}$

Este evento es descrito por Curcio con más detalle que ningún otro autor antiguo conservado. Pero al mismo tiempo el historiador romano introduce una interesante indicación metodológica advirtiendo sobre su actitud en relación con la fuente que está manejando. Antes de presentar el discurso del escita, Curcio anticipa al lector que puede haber en su relato cuestiones inverosímiles, pero que él se limita a recoger lo expresado en los textos que consulta:

Lo que, según la tradición, dijeron (los escitas) al rey puede ser que choque a nuestros espíritus y a nuestras costumbres, a quienes nos ha tocado en suerte conocer tiempos y culturas más refinados. Pero, aun suponiendo que su discurso pueda ser menospreciado, no debe serlo nuestra fidelidad histórica y expondremos, sin alterarlo lo más mínimo, lo que la tradición nos ha legado. ${ }^{9}$

En este trabajo nos proponemos plantear que, en efecto, Curcio reflejó en este pasaje la narración recogida en su fuente. No obstante, el propio autor latino habría introducido ideas personales, al tiempo que habría acortado y manipulado el original para conseguir el tono que él pretendía en su relato sobre el gran macedonio. Como veremos, Curcio carga deliberadamente las tintas en contra de Alejandro, al tiempo que adapta el lenguaje original a su propio estilo y, en cierto modo, evoca indirectamente la figura de Calígula, al igual que ocurre en otros pasajes de su obra. ${ }^{10}$ Este

6 Tuplin 2010, 290. Es interesante notar que Augusto insistió en su dominio sobre las dos orillas del Tanais: $R G 31.2$.

7 Sobre esos aspectos tácticos, véase Rickly 1975; BosworTH 1995, 22-25.

8 Alejandro pretendió haber encontrado al otro lado del río los trofeos del viaje de Dioniso, que en realidad serían túmulos de jefes escitas: Curt. 7.9.15; Bosworth 1995, 31. Sobre la relación de Alejandro con este dios, véase sobre todo GouKOWSKY 1981.

9 Curt. 7.8.11: Sic, quae locutos esse apud regem memoriae proditum est abhorrent forsitan moribus oratoribusque nostris, et tempora et ingenia cultiora sortitis. Sed, ut possit oratio eorum sperni, tamen fides nostra non debet; quae, utcumque sunt tradita, incorrupta proferemus (traducción de F. Pejenaute Rubio, Biblioteca Clásica Gredos, 1986, 390).

10 La posible relación con Calígula de las críticas de Curcio a Alejandro conduce al debate sobre la datación de este autor, posiblemente en época de Claudio, según la opinión más extendida. Para un repaso general, véase AtKinson 1980, 19-57; ID. 1994, 26-28; ID. 2000, 319; YARDLEY - HeCKel 1984, 1-4, y además CizeK 1995, 210; André 1998, 26; Dion 1998, 74; cf. Yardley - Atkinson 2009, 40; Lerouge-Cohen 2014, 203-204, n. 1. Hay, no obstante, quienes plantean fechas diversas, desde Augusto a los Severos: véase FugMANN 1995, y BAYNHAM 1998, 201-219, quien admite que el emperador bajo el que escribe Curcio "was most likely Vespasian, but on the evidence we have, we cannot be certain of his identification" (219). Para más referencias, véase ATKINSON 1998, 3451-3455; McKechnie 1999, 50-51; Von Albrecht 1999, 995; MahÉ-Simon - Tringuier 2014, $20-25$. 
episodio, por tanto, transcurre entre lo tópico, lo fantástico y lo épico, produciendo una original combinación de puntos de vista.

\section{La embajada escita: una escena tópica}

Como ya mostramos en un trabajo anterior, el encuentro de Alejandro con los embajadores escitas no hace sino reproducir una escena-cliché que se repite en diversos historiadores antiguos: hay un imperio que pretende dominar a un pueblo salvaje y pobre, pero virtuoso y aguerrido; los embajadores de éste reprochan la ambición y la codicia de los conquistadores y advierten que lucharán con todas sus fuerzas para preservar su libertad. Podríamos traer a la memoria diversos episodios similares que comenzarían ya en la obra de Heródoto: pensemos en las advertencias a Ciro el Grande por parte de Tomiris, reina de los masagetas, y las palabras de los etíopes a los emisarios de Cambises. ${ }^{11}$ Autores posteriores recogieron igualmente este tipo de escena: el encuentro entre los escitas y los enviados del rey Vezosis de Egipto, ${ }^{12}$ entre el rey escita Ateas y Filipo II de Macedonia, ${ }^{13}$ entre Dromiquetes y Lisímaco, ${ }^{14}$ entre los nabateos y Demetrio Poliorcetes, ${ }^{15}$ así como las palabras de Ariovisto, rey de los suevos, ante los enviados de Julio César. ${ }^{16}$ Ecos de este tipo de episodio podrían intuirse igualmente en el discurso del britano Cálgaco a sus tropas recogido por Tácito y, en cierto modo, cabría percibir aquí una reminiscencia del Diálogo de Melos de Tucídides, pues se trata de mostrar la razón del débil frente a la imposición del poderoso. ${ }^{17}$ Cabría, por otro lado, encontrar pasajes en el propio Curcio que evocan una libertad de palabra similar a la de estos escitas, como se ve en los discursos de Caridemo y Cobares, o que plantean el debate sobre si es o no conveniente continuar las conquistas, como ocurre con las palabras de Ceno a Alejandro en la India. ${ }^{18}$

También vemos en el pasaje de Curcio que quien habla a Alejandro es el más anciano de los escitas, reproduciendo el topos del diálogo entre un joven monarca y un sabio anciano. Se trata de una situación que podemos encontrar, por ejemplo, en el encuentro del rey con los sacerdotes de Amón, pero también en las conversaciones

11 Hdt. 1.206-207, 3.21.2-3; 4.127, respectivamente. En general, sobre este tipo de escena, véase Ballesteros Pastor 2003; Bosworth 2004.

12 Just. 2.8.12; Oros., Hist., 1.14.1-3; cf. Ivantchiк 1995.

13 Just. 9.2.1-2; Plu., Mor., 174f; Lens Tuero 1994a.

14 D.S. 21.12; Str. 7.3.8; Plu., Mor., 126 e-f, 183c, Demet. 52.4; Lens Tuero 1994b; Bosworth 1996a, $150-151$

15 D.S. 19.97.3-5; Lens Tuero 1994; Bosworth 1996a, 148-151; Ballesteros Pastor 2003, 25-27.

16 Caes., $B G, 1.36 .7$; Helmreich 1927, 214-217; Brunt 1978, 181; Ramage 2001, 158.

17 Sobre la relación entre el discurso de Cálgaco y el del escita, véase Steele 1915; Von Albrecht 1999 , 997; Baynham 1998, 88, con n. 111; Ballesteros Pastor 2003, 31-32. Sobre la analogía entre la gesta de Alejandro y la campaña de Agrícola, véase además Bosworth 2004, 555-559; Ballesteros Pastor 2011. Sobre el Diálogo de Melos (Th. 5.84-116) como modelo para estas escenas, véase MaZZARINO 1966, 339-400.

18 Véanse las críticas al poder por parte de Caridemo (Curt. 7.4.9-13) o Cobares (Curt. 7.4.9-13; BOSWORTH 1996a, 150, n. 56; Devillers 2014, 27). Sobre el discurso de Ceno (9.3.5-15), véase Tandoi 1964, 143-145; Romm 1992, 143-144, y sobre su estructura, Muckensturm-Poulle 2013. 
del macedonio con Diógenes y con los brahmanes. ${ }^{19}$ Curcio habla de una embajada de veinte personas: se trataría probablemente de un dato encontrado por nuestro autor en su fuente, que habría reflejado una cifra tan redonda que podemos considerar ficticia. ${ }^{20}$

\section{Situaciones inverosímiles: ¿un episodio interpolado en el relato?}

La secuencia narrativa de los hechos tal y como la presenta Curcio tiene una serie de incongruencias que nos llevan a pensar que estamos ante un episodio intercalado dentro de la narración. ${ }^{21}$ Son bien patentes las divergencias con Arriano, que debió haber seguido a Ptolomeo y Onesícrito en esta parte de su relato. ${ }^{22}$ Así pues, mientras que en la Anábasis no hay eco alguno de estos emisarios, en las Historiae Alexandri se habla paralelamente a esta embajada de un destacamento de jinetes escitas enviados por su rey para destruir la ciudad fundada por el Magno junto al río. ${ }^{23}$ Pero Curcio no dice explícitamente que tales escitas, a las órdenes del príncipe Cartasis, hubieran llegado a enfrentarse entonces con los macedonios. ${ }^{24}$ En efecto, poco después de aludir a estos jinetes Curcio relata con detalle la preocupación de Alejandro al contemplar desde la orilla del río a los escitas moviéndose con sus caballos por la ribera opuesta

19 Wilhelm 1928, 40; MacL. Currie 1990, 74; Ballesteros Pastor 2003, 29. Sobre el encuentro con los brahmanes, véase Plu., Alex., 64-65; Arr., An., 7.2.2-6; Ps. Callisth. B 3.6; Martin 1959; Stoneman 1995; Szalc 2011. Sobre el oráculo de Amón, véase Curt. 4.7.25; AtKInson 1980, 355-358; Prandi 1990, 365369. Para otras situaciones similares, véase por ejemplo Plb. 3.33.2; Liv. 5.36.7-8; 21.19.8; 28.39.1; 29.17.1; 34.59.1; 40.47.6; Curt. 4.11.2.

20 No es infrecuente que Curcio defina el número concreto de emisarios de una legación, y en particular es habitual la repetición del número treinta: 4.3.19; 5.3.11; 7.10.4; 8.4.23; 8.11.1; o trescientos: 6.6.4; 6.8.1; 7.11.7.

21 Ballesteros Pastor 2003, 26. No podemos asegurar si dicha interpolación se debió directamente a Curcio o a la fuente que siguió para este discurso, aunque consideramos más probable esto último. No obstante, Curcio afirma haber seguido en su obra a varios autores: véase BAYNHAM 1998, 57; YARDLEY AtKinson 2009, 19-20. El discurso escita fue atribuido a Calístenes por Helmreich 1927, 211, y a Clitarco por Wilhelm 1928, 43; Pearson 1960, 222; Atkinson 1980, 66; Baynham 1998, 79, 89. La abundancia de discursos, característica de Curcio, habría sido un punto común con las Historias Filípicas de Pompeyo Trogo: Ballesteros Pastor e.p.

22 Son citados por Arriano antes y después del combate con los escitas: An. 4.3.5; 4.6.1. Las divergencias entre Arriano y Curcio respecto a este pasaje fueron notadas por PEARson 1960, 166-168; HAMMOND 1983, 143; BoswOrTH 1995, 15-31.

23 Curt. 7.7.1. La ciudad era Alejandría Escate, de la que también hablan otras fuentes: Arr., An., 4.4.1; Curt. 7.6.25-27; Just. 12.5.12; Str. 11.11.4; Bosworth 1995, 25-27; YARdLey - Heckel 1997, 219-220.

24 El Epítome de Metz indica que este hermano del rey escita había sido enviado para "prohibir" a Alejandro cruzar, lo que podría implicar una entrevista cara a cara con el macedonio, aunque más bien se trata de un error a la hora de abreviar el relato: Scytharum imperator fratrem suum Carthasim cum copia magna misit, qui Alexandrum flumen transire prohiberet. Si prescindimos del nombre de su jefe, quizás introducido por error, estas tropas podrían identificarse con los escitas mencionados por Arriano (An. 4.3.6), que provenían del lado "asiático" del río; cf. BosworTH 1995, 22-23. Indirectamente, se sugiere que Alejandro ha sido atacado ya por los escitas como causa para cruzar el río (Curt. 7.7.11), pero esto se contradice con el contexto general de la narración (cf. 7.8.16). 
y haciendo gestos desafiantes. ${ }^{25}$ Resulta, por tanto, más creíble considerar que esta tropa al mando de Cartasis no habría formado parte de embajada alguna, si es que la hubo. En el mejor de los casos, los escitas habrían enviado a alguien para indagar las intenciones de Alejandro, pero eso no lo confirma ninguna otra fuente conservada. Arriano, que es el único aparte de Curcio en ofrecer un relato preciso de este episodio (An. 4.4.4-8), habla de un único enfrentamiento con estos escitas, que se produce cuando los macedonios cruzan el Tanais. Por lo tanto, podemos limitarnos a concluir que un destacamento de estos bárbaros habría observado a los macedonios, amenazándolos con sus gestos desde el otro lado del río. La fuente seguida por nuestro autor habría combinado diferentes tradiciones, interpolando una embajada en principio creíble, pero no verídica.

Cartasis es un nombre que, aparte de Curcio, aparece solamente en el Epitome de Metz. Como sugirió Heckel, podría relacionarse con Satraces, el líder de los escitas que lucharán contra los macedonios cuando éstos lleguen al otro lado del Tanais. ${ }^{26}$ Teniendo en cuenta que las metátesis eran muy frecuentes en la transcripción de los nombres propios iranios, podríamos plantear que el nombre de este personaje (Satraces o Sartaces) habría derivado en Cartaces y de ahí en Cartasis, quizás escrito así por la fuente de Curcio, o quizás fruto de una errata de este autor o de la posterior tradición manuscrita. ${ }^{27}$ Por lo tanto, el relato de Curcio resulta equívoco, pues Cartasis era en efecto el jefe de la tropa enviada para frenar a Alejandro, pero permaneció en todo momento en la otra orilla del río. Como ya señalamos en otro trabajo, el escita que pronuncia el discurso ante Alejandro es un personaje anónimo, y por tanto no podemos identificarlo abiertamente con el mencionado príncipe. ${ }^{28}$

Hay otro aspecto inverosímil en este episodio. Como demostró Rickly, pensar que se cosieron 12.000 pieles para el desplazamiento de los macedonios por el río resulta a todas luces excesivo. Además, según plantea Rickly, no se trataría tanto de odres (utres) como de pequeñas embarcaciones de cuero (rates), pues Curcio habría entendido mal el lenguaje de su fuente. ${ }^{29}$

Por último, llama la atención la alta elocuencia del bárbaro escita, que utiliza un lenguaje florido y lleno de artificios retóricos para recriminar y amenazar a Alejandro.

25 Curt. 7.8.1-2; Arr., An., 4.4.2-3. Sobre esta inquietud, que lleva al rey a la blasfemia y la superstición, véase Curt. 7.7.7-10; Montero Herrero 1990, 344-345.

26 Arr., An., 4.4.8; HeCKel 2006, 78-79, s.v. "Carthasis". Si seguimos a Arriano, habría que identificar a este personaje con el que se entrevista más tarde con Alejandro (An. 4.15.1-3).

27 Por citar sólo algunos ejemplos de estas metátesis, recuérdese que Heródoto llama Artafrenes a Artafernes, y que hay una confusión entre Oxiartes y Oxiatres, padre de Roxana: véase LECOQ 1986, 652; HeCKel 2006, 186 s.v. "Oxatres".

28 Ballesteros Pastor 2003, 26.

29 Curt. 7.8.7; Rickly 1975; Bosworth 1995, 27-28. Odres hinchados, a modo de flotadores, están documentados desde los relieves asirios para el cruce de los ríos por las tropas. Curcio (7.9) y Arriano (4.4.45) discrepan respecto al uso de catapultas: el primero sugiere que se usaron para proteger a los macedonios mientras cruzaban y fueron transportadas al otro lado del río, pero Arriano se limita a hablar de su empleo antes de que estas tropas comenzaran a cruzar. Bosworth 1995, 28-29, sugiere que la versión de Curcio habría sido la correcta. No obstante, éste (7.9.1-7) no habla del uso de catapultas sobre balsas en el mismo río, como sugiere Bosworth, sino sólo de los disparos de éstas. Hay que tener en cuenta el problema de la fuerte corriente que describe Curcio, 7.9.6-7. Desacreditando la versión del autor latino, cf. RickLY 1975, 36. 
Esto ya resultó insólito a Curcio, que incluyó antes del discurso la observación preliminar que hemos mencionado. ${ }^{30}$ Sin entrar de momento a valorar este aspecto, debemos tener en cuenta que la elocuencia bárbara era una licencia admitida por la historiografía antigua. No analizaremos una por una las entrevistas entre diferentes pueblos que hemos comentado arriba, ni muchos otros ejemplos que se encuentran en la literatura clásica, pero podríamos traer a nuestra memoria un pasaje de Diodoro Sículo en el que, a propósito de Viriato, se explica y justifica la capacidad retórica de los pueblos primitivos. ${ }^{31}$ Sin embargo, hay que tener en cuenta que Diodoro habla de una expresión sencilla, ruda y directa, mientras que Curcio plantea un pasaje erudito, lleno de sentencias latinas y con todo tipo de recursos oratorios.

\section{Un escita demasiado instruido. Reflexiones sobre la fuente de Curcio}

Por más fieles que pretendan ser, los discursos en la historiografía antigua son siempre fruto de reelaboraciones del autor. Pero esto era algo aceptado en general, mientras que hay cuestiones específicas que atañen al pasaje que nos ocupa: más que la elocuencia de un bárbaro, que como hemos dicho era una licencia admitida, lo que verdaderamente llama la atención son las referencias cultas que aparecen en el discurso escita. Hay diversas alusiones a tradiciones escitas que aparecían recogidas en la obra de Heródoto, pero lo que nos resulta más sorprendente es la frase donde el emisario dice saber bien que "los proverbios griegos se burlan de los desiertos escitas". ${ }^{32}$ Buscando referencias en la tradición literaria griega a los "desiertos" habitados por este pueblo, las únicas burlas explícitas estarían presentes en un pasaje de Los Acarnienses de Aristófanes (702-3). Sin embargo, puesto que Curcio habla de un proverbio, quizás se esté refiriendo a un acertijo que aparece en Las Aves, en donde se hace burla del frío que pasan los escitas errantes. Pero estos versos no eran originales de Aristófanes, sino que habrían estado tomados de Píndaro, según informa el escoliasta del comediógrafo. ${ }^{33}$ Es por tanto probable que el pasaje del célebre poeta griego sobre los escitas continuara en versos que Aristófanes no recogió, donde se haría referencia explícita a los desiertos, o que la fuente de Curcio se refiriese de un modo general a la burla que hace Píndaro del entorno donde los escitas vivían.

Resulta ciertamente inverosímil que la poesía de Píndaro o los ingeniosos textos de Aristófanes hubieran llegado hasta el confín de Asia. Quizás a Curcio esta referencia le resultó sorprendente, pero prefirió reproducirla, quizás de forma un tanto mecáni-

\footnotetext{
30 Vide supra $\mathrm{n} .9$.

31 D.S. 33.7.3, 7; Ballesteros PAstor 2009, 36. La rudeza en la expresión era un atributo bárbaro: Dauge 1981, 648-653.

32 Curt. 7.8.23: Scytharum solitudines graecis etiam proverbiis audio eludi. Para las referencias en este discurso a información transmitida por Heródoto, véase WilHelm 1928, 35-47; BosworTh 1996a, 148-151; Baynham 1998, 123; Ballesteros Pastor 2003, passim. El término "desierto" es un tópico en la descripción de territorios bárbaros, y en particular se aplica frecuentemente a los escitas: KolEndo 1991; BALlesteros PASTOR 2013, 287.

33 Ar., $A u ., 941-945=$ Pind. fr.105b Snell; Schol. Ar., $A u$., 941-945; cf. Rogers 1927, 221, nota d. Para un análisis de este pasaje de Píndaro y su contexto, véase Morgan 2015, 322-324.
} 
ca. Lo que esta alusión literaria revelaría es que la fuente del discurso de Curcio es un autor griego o un bárbaro helenizado, y que su amor por la verdad, en lo que a la embajada escita se refiere, es relativo. ${ }^{34}$ Es este anónimo escritor quien conoce esos versos, y se permite una licencia al demostrar que él (y no precisamente el embajador escita) tiene un profundo conocimiento de la literatura helénica.

\section{Unos bárbaros no tan virtuosos: la elección de Curcio ante discursos pareados}

La escena de la embajada escita contiene una breve respuesta de Alejandro tras las palabras del anciano emisario. Con un escueto párrafo, el rey se limita a decir que toma nota de los consejos recibidos y anuncia que actuará siguiendo su propia fortuna. Según nuestro criterio, la fuente de Curcio habría recogido discursos pareados, en los que a la disertación del escita habría correspondido la réplica del rey, más o menos equiparable en extensión. Este tipo de construcciones retóricas son muy características de las descripciones de embajadas, en donde se da un intercambio de argumentos entre una y otra parte, es decir, entre los emisarios y quienes reciben a éstos. Curcio habría resumido la intervención de Alejandro en unas pocas líneas para dar más realce a las críticas hacia el macedonio. ${ }^{35}$ La respuesta de Alejandro que Curcio habría encontrado en su fuente habría estado probablemente en consonancia con el tono de este episodio de las Historiae Alexandri, en donde, lejos de manifestar una perspectiva abiertamente negativa del rey, éste aparece como un caudillo audaz, valeroso y clemente. En verdad resulta bastante significativo que tras una censura tan acerba como la que se hace a Alejandro en el discurso escita, se describa positivamente la posterior victoria macedonia, una vez cruzado el río, con muestras de arrojo y de inteligencia a un tiempo. Según Curcio, Alejandro se muestra compasivo con los vencidos y manifiesta que su único afán al combatir era la gloria, antes que el deseo de realizar una demostración de fuerza. ${ }^{36}$

Consideramos, pues, bastante plausible que el tono de la escena que presentaba la embajada escita habría sido menos desfavorable a Alejandro que el transmitido por nuestro biógrafo. Las palabras del rey habrían sido pronunciadas después de las duras acusaciones de los escitas, con lo que la réplica del aquél quedaría en cierto modo como argumento definitivo. Pensemos de nuevo en el discurso de Cálgaco, al que siguen las palabras de Agrícola llenas de honor y dignidad. ${ }^{37}$ No es que Tácito no

34 Sobre las fuentes de Curcio, vide supra n. 2.

35 Sobre los discursos pareados en Curcio, véase BAyнnam 1998, 46-47, y para esta parte de las Historiae Alexandri, Devillers 2014, 11; ID. 2014a, 121-122 (que prefiere contrastar el discurso escita con las anteriores palabras de Alejandro en 7.7.10-19). Para otros ejemplos en los historiadores latinos, véase KRAUS - WoOdMAN 1997, 13, 19, 60. El recurso de reducir una de las intervenciones en discursos pareados es frecuente en Justino: véase BALlesteros PASTOR 2009a.

36 Curt. 7.9.18; Ballesteros Pastor 2011, 45. La búsqueda de gloria antes que de dominio aparece igualmente en Justino, 1.1.7; 2.3.7. Según DeviLlers 2014, 8, se trataría de una perspectiva común a los libros VI y VII de Curcio: el peligro viene de los enemigos, ya dispuestos al combate, mientras que la victoria es un arma de disuasión para incitar a los pueblos extranjeros a la paz (Curt. 6.3.5; 7.7.13).

37 Tac., Agr., 30-34; Ballesteros Pastor 2013, 63. 
hubiera puesto énfasis en las ideas del britano, pero en definitiva es Agrícola el que contrapesa las duras críticas que se han hecho a Roma.

Esta imagen positiva de Alejandro se vendría a corresponder con una interpretación no tan virtuosa de la actitud de los emisarios. Como el propio Curcio recoge, los escitas eran tenidos como encarnación del ideal del "buen salvaje" que aún no ha sido corrompido por la civilización. ${ }^{38}$ Pero esa imagen no se corresponde por completo con la escena que estamos estudiando. En efecto, en el discurso se habla de virtud, de valor, de sobriedad, pero al mismo tiempo la actitud de estos escitas podría ser abiertamente calificada como una demostración de insolencia bárbara. ${ }^{39}$ Habían comenzado los emisarios clavando sus miradas en el rostro del rey y burlándose de la baja estatura de éste, para después amenazarlo, presumiendo de pasadas proezas que, en la mayoría de los casos, tampoco habrían sido realizadas por aquellos remotos pueblos del Iaxartes..$^{40}$ Creemos que aparecería aquí una perspectiva tal vez próxima al mundo iranio, pues mientras los escitas se habían convertido para algunos en un arquetipo de raza virtuosa, hay narraciones en las que éstos son duramente criticados: recordemos al ambicioso y frívolo escita que aparece en la Ciropedia de Jenofonte. Pero también existía entre los griegos una imagen de los escitas como feroces y sanguinarios que ya estaba presente en la leyenda de Ifigenia en Táuride, y alcanzaría hasta autores como Estrabón y Ovidio. ${ }^{41}$

En este sentido, resulta útil comparar todo el pasaje de Curcio sobre el combate más allá del río con la descripción que hace Arriano. El autor de Nicomedia insiste en que Alejandro no ha derrotado al pueblo escita, sino únicamente a una horda de bandidos. Ello supone salvaguardar el carácter invicto de los escitas, reafirmando que resistieron a las armas de los macedonios, según aparece explícitamente en el Epitome de Justino. ${ }^{42}$ Quizás podríamos considerar que Arriano hubiera tomado este pasaje de Onesícrito quien, como seguidor de la filosofía cínica, habría insistido en las virtudes del pueblo escita. Curcio, por el contrario, describe abiertamente el triunfo de las tropas de Alejandro y la clemencia de éste hacia los enemigos vencidos. ${ }^{43}$

38 Véase sobre todo la valoración positiva de los escitas abios, a los que Alejandro ha recibido poco antes: Curt. 7.6.11. Para más referencias, véase en general Lovejoy - BoAs 1935, 315-344; HARTOG 1980, 181 -188 y passim; Dauge 1981, 181-188; Lévy 1981; Baynham 1998, 88; Ballesteros Pastor 2003, 28.

39 Tal es la perspectiva de BRUNT 1978, 181, respecto a la actitud de Ariovisto (Caes., $B G, 1.36 .7$ ).

40 Sobre la estatura del rey: Curt. 7.8.9; cf. Ps.Callisth. B 2.15, 3.4. Éste era un elemento asociado a la majestad regia: véase por ejemplo Curt. 6.5.29; Hdt. 9.96.2; 7.187.2; X. Cyr. 8.1.40-43; Plu., Rom., 3.4; App., Syr., 57, Lyb., 106, Mith., 112; Sall., Hist., fr.2.77M; Vir.Ill. 76.1. Curcio había insistido en la corpulencia de los escitas: 4.13.5; 7.4.6. La alusión a la victoria escita sobre los asirios (Curt.7.8.18) parece fruto del afán por reproducir el tópico de la translatio imperii, igual que aparece en Diodoro 2.48.5: cf. Just. 2.3.1-4; Ballesteros Pastor 2003, 30-31.

41 X., Cyr., 8.10.25-50. Sobre ese aspecto del mito de Ifigenia, véase por ejemplo KyriaKou 2006, $34-$ 36, 135-136, 164-165 y passim. Sobre Estrabón, véase CoRDE 2005. Sobre Ovidio, véase por ejemplo Trist., 1.8.40; 4.6.47; Pont., 1.7.9; 2.1.65; 2.2.110. Véase también Hor., CS, 53, Carm., 1.35.9-10; Amm. 23.6.60.

42 Arr., An., 4.5.1; Bosworth 1995, 31-32; cf. Just. 2.3.1; Ballesteros Pastor 2013, 6-12, 139-141.

43 Curt. 7.9.17: invictos Scythas esse crediderant. Poco antes Alejandro ha recibido sendas embajadas de los escitas "europeos" (probablemente ribereños del Iaxartes) y de los escitas abios (pueblo citado por Homero), y el rey aparece dándoles órdenes (Arr., An., 4.1.1; Curt. 7.6.11-12; cf. 9.2.33). No obstante, se ha relacionado el discurso escita de Curcio con la tradición cínica: André 1990, 21; MACL. CurRIE 1990, 74. Sobre los escitas y su relación con la filosofía cínica, véase MARTIN 1996. 


\section{6. ¿Alusiones indirectas a Calígula en el discurso escita?}

La existencia o no de alusiones a Calígula dentro de la obra de Curcio sigue siendo una cuestión controvertida para los investigadores. En la base del debate estaría el problema de la datación de esta biografía, pues se han propuesto opciones que irían desde la época de Augusto hasta la de los Severos. No obstante, la tendencia más relevante en la actualidad es la de situar a Curcio en el reinado de Claudio, y considerar que algunas de las críticas de este historiador hacia Alejandro habrían sido en realidad traslaciones de la censura a determinadas facetas del reinado de Calígula. ${ }^{44}$ Así, centrándonos exclusivamente en el libro X de las Historias de Alejandro, Yardley y Atkinson recogen no menos de once posibles referencias a este emperador romano. ${ }^{45}$

Por nuestra parte, consideramos que el discurso escita contiene algunas frases que podrían ser interpretadas como una alusión indirecta a episodios de la vida de Calígula que fueron objeto de censura por diversos autores antiguos. En primer lugar mencionaremos el triunfo del emperador sobre el golfo de Bayas, desfilando sobre un puente de barcas que se hizo construir para la ocasión. Dicho puente fue entendido como una imitación del construido por Jerjes para cruzar el Helesponto, evocando un paso desde Asia a Europa y viceversa, pero también fue relacionado precisamente con el puente realizado por Darío para su invasión de la Escitia europea. ${ }^{46}$ Se trataría, pues, de la misma situación en la que se encuentra Alejandro en nuestro pasaje, ya que considera hallarse en el límite entre los dos continentes. Como afirma Curcio, el macedonio tiene de nuevo Europa al alcance de la mano, y puede trasladarse fácilmente de un continente a otro, lo que nos evoca más bien el desfile de Calígula que la táctica militar de Alejandro, que hubo de construir catapultas y balsas para posibilitar el paso de sus tropas por el río, evitando las armas enemigas. ${ }^{47}$ Pero al mismo tiempo, el emperador romano trató abiertamente de imitar a Alejandro al atravesar el puente de Bayas: como afirmó Kleijwegt, el hecho de realizar el puente a imitación de la Vía Apia, el llevar en el desfile a un rehén llamado Darío y el vestir la coraza que, presuntamente, había pertenecido a Alejandro, reflejaría un afán de Calígula por describir un triunfo sobre Oriente, emulando las hazañas del gran macedonio. ${ }^{48} \mathrm{De}$ hecho, Pompeyo Magno había hecho antes algo similar en el triunfo tras su campaña

44 En torno a este debate, vide supra n. 10. Sobre la imitatio Alexandri de Calígula, véase BRUHL 1930, 211213; Heuss 1954, 85-87; WIRTH 1976, 186; CROISILle 1990, passim.

45 Curt. 10.1.7; 2.12; 5.14; 5.33; 5.34; 5.36; 6.4; 6.15; 7.11; 9.3-6; 9.16; YARdLeY-Atkinson 2009, ad loc., así como 43, n. 123. Véase además Curt. 8.5.14-19; 8.7.1-6.

46 Suet., Cal., 19, cf. 52.3; D.C. 59.17; I. AI 19.5-6; Sen., Brev.Vit., 18.5; cf. Hurley 1993, 76-78; WARdLE 1994, 195; Malloch 2001, 207-209; Montero Herrero 2012, 138-140.

47 Curt. 7.8.13: Ab Europa petis Asiam, ex Asia transis in Europam; 7.8.12: altera manu Orientem, altera Occidentem contingeres; cf. 7.7.14: Et quantum aestimandum est (...) quae tam longo intervallo Natura videtur diremisse, una victoria subito committere?

48 Kleijwegt 1994. Malloch 2001, piensa en la procesión báquica de Alejandro en el 325 a.C. (D.S. 17.106.1; Curt. 9.10.24; Arr., An., 6.28.1). Woods 2002, propone que Calígula estaba probando la viabilidad de construir un puente sobre el Canal de la Mancha. Esta coraza habría sido tomada de la tumba de Alejandro, aunque no tenemos noticia de que Calígula hubiera visitado Alejandría siendo adulto: Erskine 2002, 178. 
oriental (61 a.C.), vistiendo un manto que supuestamente había sido de Alejandro, y llevando en su cortejo a un presunto rey de Media llamado igualmente Darío. ${ }^{49}$

Otra frase del discurso escita aludiría al triunfo sobre las fuerzas de la Naturaleza: "Cuando termines con el género humano, te tendrás que batir con los ríos, los bosques y las fieras salvajes". ${ }^{50}$ Es cierto que Alejandro se mostró en diversas ocasiones como vencedor sobre los Elementos, pero también podríamos pensar aquí en una evocación de aspectos de la vida de Calígula. ${ }^{51}$ En primer lugar, el puente de Bayas fue considerado una alteración de las leyes de la Naturaleza, pues en palabras de Dión Casio (59.17.9) el emperador quiso hacer el día noche igual que había hecho tierra el mar. También Calígula pretendió entonces estar caminando sobre las aguas, igual que también se decía que había hecho Alejandro. ${ }^{52}$ En segundo lugar, podríamos evocar la campaña de Calígula en Germania, cuando éste se lanza con parte de la caballería pretoriana a luchar contra un bosque porque, según Suetonio, no tenía enemigo con el que enfrentarse ${ }^{53} \mathrm{El}$ emperador habría tratado de manifestar entonces un triunfo sobre las fuerzas de la Naturaleza, puesto que ordenó adornar los árboles como si fueran trofeos, y decretó para sus hombres una "corona exploratoria" que llevaba el sol, la luna y las estrellas. ${ }^{54}$ Ello concordaría además con otros episodios en los que Calígula habría pretendido mostrarse como dominador de los Elementos, que de nuevo podríamos relacionar con Jerjes y su canal en el Monte Atos. ${ }^{55}$ Pero en particular, habría que hacer referencia al peculiar combate con el mar que el emperador ordenó, y que aparece ridiculizado en las fuentes antiguas. ${ }^{56}$ Este episodio también podría interpretarse como una evocación de la gesta de Alejandro, que al llegar al océano en Gedrosia ofreció un sacrificio a Poseidón. ${ }^{57}$

49 App., Mithr., 106, 117; D.S. 40.4. Sobre este triunfo como un episodio de imitatio Alexandri, véase Bellen 1987; Spencer 2002, 19. Woods 2006/7, defiende que en realidad Calígula estaría imitando a Pompeyo en el centenario de su triunfo del 61 a.C., aunque creemos que es solo una coincidencia, ya que el emperador trataba de exaltar sus actividades en Germania y su combate contra el mar. Algunos han creído que Calígula vistió en este desfile el mismo manto que Pompeyo: WeInstock 1971, 38, n. 8; Woods 2006/7, 125-127.

50 Curt. 7.8.13: Si humanum genus omne superaveris, cum silvis et nivibus et fluminibus ferisque bestiis gesturus es bellum; cf. Sen., Nat.Quaest., 5.8.10; Tac., Agr., 30.3-4; Flor., Epit., 1.45.60; Plu., Pyrr., 12.3-5.

51 Alejandro doma a Bucéfalo, calma el mar y controla la corriente de los ríos: en general, véase DE Polignac 1996; Malloch 2001a, 554.

52 D.C. 59.17.7; cf. 59.26.6; I. A.I.19.5-6; Montero Herrero 2012, 139-140. Sobre Alejandro, véase DeSNIER 1995, 27-40; BOUVIER 2004.

53 Suet., Cal., 45.1: deficiente belli materia. Sobre la campaña germánica de Calígula, véase Suet., Cal., $43-$ 48, 51.2-3, Galba, 6.3; Tac., Ger., 37.5, Hist., 4.15; D.C. 59.21.2-3; Eutr. 7.12.2; Barrett 1989, 125-135; WoOds 2012, 444-452.

54 Suet., Cal., 45.1; PiCARD 1957, 328; cf. contra WardLe 1994, 310. Las estrellas eran también un motivo relacionado con Alejandro: véase HanNESTAD 1997, 63. Sobre las estrellas en la propaganda de Calígula véase Suet., Cal., 45.1; Weinstock 1971, 381-384; WardLe 1994, 150.

55 Suet., Cal., 37.3; Hurley 1993, 143. La referencia a allanar montes y elevar el fondo del mar era un topos en la literatura romana: cf. por ejemplo Sall., Cat., 13.1; Just. 2.10-24; WARdLE 1994, 282-3. Sobre la relación entre el gobierno de Calígula y el despotismo persa, véase además Sen., Ben., 2.12.2; MALLOCH 2001, 207-209. Estas palabras de Suetonio han sido relacionadas también con la construcción de un faro en la Galia: D’Erce 1966; Woods 2002, 165. Calígula planeó una ciudad en la cima de los Alpes: Suet., Cal., 21, cf. 37.3; WARDLE 1994, 201.

56 Suet., Cal., 46; D.C. 59.21.3, 59.25.1-3; Aur.Vict., Epit., 3.11-12.

57 Montero Herrero 2012, 50. Respecto a Calígula véase además Barrett 1989, 135-138; Woods 2000; HIND 2003. 
Aunque el topos del diálogo entre anciano y joven rey se repite en numerosos relatos antiguos, se ha detectado una analogía entre la embajada escita y el encuentro de Alejandro con Diógenes, que a su vez ha sido relacionado con la entrevista entre Calígula y Demetrio el Cínico. ${ }^{58}$ Del mismo modo, la reiteración en el discurso escita de expresiones escritas por Séneca el Rétor y su hijo para criticar a Calígula podría reflejar de qué manera Curcio tenía presente al denostado emperador romano. ${ }^{59}$

\section{El dilema de Alejandro y las fronteras del imperio romano}

Hay otro asunto implícito en el discurso escita, que es el debate sobre la conveniencia de continuar o no las conquistas. Éste debió ser un tema importante para Curcio, que no sólo describe los riesgos del cruce del Tanais, sino que después vuelve sobre este asunto ya en la India, cuando Ceno pronuncia un discurso en el que insta a Alejandro a detener el avance hacia el Este. Tal dilema está presente entre los romanos desde la debacle de Craso en el 53 a.C., pero más intensamente a partir del desastre de Varo en Teutoburgo, ya bajo el principado de Augusto. ${ }^{60}$

Las dificultades de la expansión del imperio romano y la problemática que ello comportaba fueron asuntos presentes bajo el reinado de Claudio. Éste fue, de hecho, uno de los emperadores que más ampliaron los dominios de Roma. No sólo deberíamos resaltar la trascendencia de su campaña en Britania, sino también su éxito frente a las tribus escitas de la región del Mar de Azov, precisamente más allá del río Tanais. Claudio, según Tácito, tuvo serias dudas sobre la conveniencia de acometer un enfrentamiento contra gentes fieras y en tierras ignotas. ${ }^{61}$ Además, este emperador anexionó Tracia, luchando contra pueblos a los que Alejandro había combatido, y que algunos, como Curcio, relacionaron con los escitas. ${ }^{62}$ De hecho, hubo generales

58 Wilhelm 1928, 45, 50; Ballesteros Pastor 2003, 29, n. 27. Sobre Calígula y Demetrio (Sen., Ep., 91.19; De Ben., 2.21), véase en particular DudLey 1937, 125-6; JerpHAGNON 1981, 58.

59 Sobre la influencia de estos autores en Curcio, véase AtKInSON 1980, 40-41; BAYNHAM 1998, 27-30; cf. Hamilton 1988. En particular, sobre este episodio, Wilhelm 1928, 48, relaciona la censura a Calígula por Séneca el Joven con Curt. 7.8.26: Denique, si deus es, tribuere mortalibus beneficia debes, non sua eripere. BARDON 1947, 125, compara Sen., Suas., 1.5: orbis illum suus non capit; con Curt. 7.8.2: orbis te non caperet. Véase además la relación entre Curt. 7.8.19 y Sen., Ben., 1.13.1, Nat.Quaest. 2. praef. 5; Lucan., Phars., 10.21; y Curt. 7.8.13 con Sen., Nat.Quaest. 5.18.10; cf. Helmreich 1927, 215; Wilhelm 1928, 43; Bruhl 1930, 213; Lassandro 1984, 166; GALE 2007, 113.

60 En general, sobre el debate acerca de la expansión del Imperio, véanse Cornell 1993; BALLESTERos PAStor 2003, 36-37 (con bibliografía).

61 Tac., Ann., 12.30; Ballesteros Pastor 2011. Sobre Britania, véase Melmoux 1990; Richard 1998; BARRETT 2000. Sobre el discurso de Ceno, vide supra n. 18. Es interesante relacionar la clemencia de Alejandro con los escitas y la mostrada por Claudio respecto al sublevado Mitrídates VIII del Bósforo: Curt. 7.9.18; Tac., Ann., 12.11.2; Ballesteros Pastor 2011, 45.

62 En general, véase KolENDo 1998. Era frecuente en la antigüedad confundir a los escitas, tracios y sármatas que vivían en torno a la desembocadura del Danubio. Curcio (10.1.44) llama getas a los escitas que derrotan a Zopirión, general de Alejandro (cf. Just. 2.3.4; 12.1.4; 37.3.2). Curcio también confunde a sármatas y escitas (7.7.3; 10.1.44). Para otros ejemplos, véanse entre otros Hdt. 4.117; Th. 2.96; Hippoc., Aër., 17-19; Lucan., Phars., 5.440; Lucian., Macr., 10; Flor., Epit., 1.39.6; Arr., An., 1.3.2; Curt. 10.1.44; Str. 7.3.2; 7.3.12; 11.2.1; Ovid., Trist., 3.11.55; 3.14.47; 4.6.47; Sordi 1990, 394-395; ANDruKh 2000; Ballesteros Pastor 2011, 44, n. 5; ID. 2013, 143-144 (con bibliografia). 
romanos que intervinieron en esa región y trataron por ello de aparecer como imitadores del gran macedonio. ${ }^{63}$

Por otro lado, el ejemplo de Alejandro en los confines de Oriente representaba una referencia para las hazañas romanas en Germania: recordemos en tal sentido que Germánico trató de aparecer como emulador de Alejandro por sus victorias en el Norte. Albinovano Pedo escribió sobre esta campaña unos versos épicos que, según la opinión general, debieron haber influido sobre la perspectiva de Quinto Curcio. ${ }^{64}$ Los germanos eran considerados por algunos autores como pueblos aguerridos que compartían ciertas virtudes con los escitas. Germania, además, estaba igualmente situada en los confines de la ecúmene. ${ }^{65}$

Por su parte, Claudio trató de exaltar su conquista de Britania como un triunfo sobre el Océano, que había llevado el poder de Roma a los límites del mundo conocido. ${ }^{66}$ Además, en Oriente tuvo este emperador que contener a los partos, a los que Curcio y otros autores consideraron descendientes de los escitas. ${ }^{67}$ En definitiva, el dilema que el anciano escita plantea a Alejandro es similar al que se debatía en la época imperial, y de un modo particular bajo Claudio: es necesario calcular los riesgos y el posible beneficio, no actuar por ambición y precaverse frente al espíritu belicoso de los bárbaros. ${ }^{68}$

\section{Conclusión}

Resulta evidente que Curcio habría manipulado el texto que empleó como fuente para este pasaje. ${ }^{69}$ La extraordinaria proeza de Alejandro es ensombrecida por críticas

63 Por ejemplo, el padre de Augusto (Suet., Aug., 94.5) y L. Calpurnio Pisón, cos. 15 a.C. (Anth.Pal. 6.335, 9.552): KienAst 1969, 433-434; Coppola 1999; cf. AndRÉ 1990, 12. Sobre las campañas de Alejandro en Tracia, véase sobre todo Arr., An., 1.1.3-4.8; Plu., Alex., 11.4-6; Str. 7.3.8; Bosworth 1980, 54-73; ID. 1996, 37-47; HAMMOND 1992, 77-83.

64 AtKinson 1980, 38; Baynham 1998, 30. El poema de Albinovano describe a Germania como fin del mundo: THÉBERT 1995, 230-231. Sobre la vinculación con Alejandro de las gestas romanas en este territorio y en Britania, véase SÁnchez León 1990, 92-95; Braccesi 1991; ID. 2006, 142-157. Sobre Germánico y Alejandro, véase además Gissel 2001.

65 Sobre esta imagen de los escitas vide supra n. 38. Sobre los germanos: Caes., BG, 1.36.6-7, 6.21-23; Tac., Ger., 5.2-3, 35; Sen., De Prou., 4.14-15; cf. Str. 7.1.3; Dupont 1995, 207-214; Marco Simón 2000. Para alusiones a la virtud común de ambos pueblos: Hor., Od., 3.24.9-11; Sen., De ira, 2.15.1; Lucan., Phars., 7.433-435. Sobre las analogías en la descripción de los bárbaros por Curcio y Tácito, véase LuND 1987, 54-56.

66 Melmoux 1990; Richard 1998; Barrett 2000.

67 Sobre la figura de Alejandro como evocadora de los problemas de Roma en Oriente, véase SIDARI 1982, André 1990, 22; Melmoux 1990; Plácido 1990, 67; SÁnchez León 1990, 96-99; Ballesteros Pastor 2003, 36, n. 67; Ndiaye 2011, 46-47; Muckensturm-Poulle 2013, 272-273; Lerouge-Cohen 2014. Sobre las vicisitudes de Claudio en la frontera con Partia, véase Levick 1990, 158-160; Frézouls 1995, 491-495; OsGood 2011, 238-240. Sobre los escitas como ancestros de los partos: Curt. 4.12.11, 6.2.14; Iust. 2.1.3, 2.3.6; 41.1.1-2; cf. Arr., Parth., (FGrHist 56 F30); Str. 11.9.3.

68 Tac., Ann., 12.20.1; cf. D.C. 37.3.2; Ballesteros Pastor 2011, 44. No se trataría tanto de plantear una imitatio Alexandri por parte de Claudio, sino de poner de relieve cómo el ejemplo de la gesta alejandrina podía suscitar motivos de reflexión durante el reinado de este emperador.

69 Curcio muestra así tener un criterio propio, independiente de sus fuentes, como subrayó THERASSE 1973. Sobre las modificaciones en el texto de los discursos por parte de Curcio, véase BAYNHAM 1998, 48-54, y en 
agrias que representan uno de los momentos más relevantes en la descripción negativa del macedonio. La fuente de Curcio habría interpolado dos discursos pareados en el relato de un combate glorioso para Alejandro, con el fin de dar realce al episodio, y por supuesto pensando en imprimir una altura retórica a la biografía del rey. Curcio, centrado en la crítica al Magno, habría dado una gran notoriedad a las palabras del escita, reduciendo la réplica del rey a un par de frases. De este modo, Alejandro aparecía duramente censurado, y su respuesta aparecía como una declaración parca y sin argumentos. Mientras tanto, sorprendentemente, las Historiae Alexandri exaltan a continuación el valor del rey, su audacia y su generosidad con los escitas vencidos. El gran conquistador ha llegado a los confines alcanzados por Dioniso.

Quinto Curcio habría reproducido básicamente el texto original del discurso escita, respetando las ideas que encontró en su fuente, pero al mismo tiempo adornó este pasaje retórico con epigramas inspirados en Séneca, que en buena medida evocarían al lector la figura de Calígula. El discurso escita convenía a la parada del Emperador romano sobre el golfo de Bayas, y quizás a otros episodios de la vida de éste: eso hizo aún más aconsejable cargar las tintas sobre las palabras del escita y minimizar en lo posible la hazaña de Alejandro quien, según los demás relatos conservados, consideró esta aventura en el Tanais como uno de los puntos culminantes de su gesta conquistadora.

La advertencia que hace Curcio al lector antes del discurso escita se encuentra pues justificada: resulta incongruente la altura retórica de las palabras de ese bárbaro, y es a todas luces impensable que conociera la literatura griega. Sin embargo, también Curcio está de manera implícita tratando de disculparse ante el lector por haber manipulado sus fuentes. Estaríamos pues ante una excusatio non petita que también, por su parte, se había convertido en una suerte de lugar común en la historiografía clásica. $^{70}$

\section{Bibliografía}

Albrecht, M. von (1999): Historia de la Literatura romana, Barcelona.

AmeLing, W. (1987): “Alexander und Achilleus”, [en] Will - Heinrichs (Hgg.), 1987, vol. II, 677-692.

particular 54: "Curtius was clearly uninterested in reproducing or trying to aproxímate for historical purposes what was actually said. (...) In short, the purpose of the speeches is artistic: they are outward, dramatic manifestations of political and literary themes". En general, sobre los métodos de Curcio, véase además Ibíd., 85-100; EAD. 2009; ATKINSON 2000.

70 Se ha comparado la frase Curcio (7.8.11) con la observación de Justino (38.3.11) manifestando que ha copiado literalmente el discurso de Mitrídates. También se han detectado relaciones con Heródoto (2.123.1, 2.130.2, 3.80.1, 4.195.2, 7.52.3), Arriano (An., 2.12.8, 7.15.6), Tácito (Hist., 2.50.2) y con el propio Curcio (9.1.34): BARDON 1947, 131; BLÄNSDORF 1971, 12; BOSWORTH 1996a, 150-151, n. 58; BAYNHAM 1998, 86; BALlesteros PAStor 2009, 34; ID. 2013, 221-222 (con bibliografía). Para las observaciones sobre la literalidad del discurso de Alejandro a Ceno, véase Curt. 9.2.12; Arr., An., 5.25.2; 5.27.1; Muckensturm-Poulle 2013, 260. 
ANDRÉ, J. M.

(1990): “Alexandre le Grand, modèle et repoussoir du prince”, [en] Croisille (éd.), 1990, 11-24.

(1998): “Sénèque et l'Historiographie de Claude”, [en] Burnand - Le Bohec-Martin (éd.), 1998, 23-39.

Andrukh, S. I. (2000): “The Scythians mixed with the Thracians (Apoll.Rhod. IV 320)”, VDI 234, 63-73 (en ruso, resumen en inglés).

Asheri, D. - Lloyd, A. B. - Corcella, A. (2007): A Commentary on Herodotus Books I-IV, Oxford.

AtKinson, J. E.

(1980): A Historical Commentary on Q. Curtius Rufus' Historiae Alexandri Magni books 3 and 4, Amsterdam.

(1994): A Historical Commentary on Q. Curtius Rufus' Historiae Alexandri Magni books 5 to 7.2, Amsterdam.

(1998): “Q. Curtius Rufus' 'Historiae Alexandri Magni'”, ANRW II 34.4, 3447-3483.

(2000): "Originality and its Limits in the Alexander Sources of the Early Empire", [en] A. B. Bosworth - E. J. Baynham (eds.), Alexander the Great in Fact and Fiction, Oxford, 306-325.

Ballesteros Pastor, L.

(2003): "Le discours du Scythe à Alexandre le Grand (Quinte-Curce 7.8.12-30)", RhMus $146,23-37$.

(2009): “Aproximación al estudio de los discursos en el Epítome de Justino", TaliaDixit 3, 29-42.

(2009a): "Pompeyo Trogo, Justino y las críticas a Roma: a propósito del discurso etolio (Iust. XXVIII 2)", MedAnt 12, 381-392.

(2011): "Beyond the Tanais: Tacitus and Quintus Curtius", $A W E$ 10, $43-48$ (http://dx.doi. org/10.2143/AWE.10.0.2141814).

(2013): Pompeyo Trogo, Justino y Mitrídates. Comentario al Epítome de las Historias Filípicas (37,1,6-38,8,1), (=Spudasmata 154), Hildesheim-Zürich-New York.

(e.p.): "The Speeches in Justin's corpusculum florum. Selection and Manipulation of Trogus' Historiae Philippicae", [en] J. C. Iglesias-Zoido - V. Pineda (eds.), Anthologies of Historiographical Speeches from Antiquity to the Renaissance, Leiden-Boston-Tokyo.

BARDON, H. (1947): “Quinte-Curce historien”, LEC 15, 120-137.

BARRETt, A. A.

(1989): Caligula. The Corruption of Power, London-New York.

(2000): "The Laus Caesaris: its History and its Place in Latin Literature", Latomus 59, 596-606.

BAYNHAM, E. J.

(1998): Alexander the Great. The Unique History of Quintus Curtius, Ann Arbor.

(2009): 'Barbarians I: Quintus Curtius' and other Roman historians' reception of Alexander", [en] A. Feldherr (ed.), The Cambridge Companion to the Roman Historians, Cambridge, 288-300.

Bellen, H. (1987): "Das Weltreich Alexanders des Grossen als Tropaion im Triumphzug des Cn. Pompeius Mangnus (61 v.Chr.)", [en] Will - Heinrichs (Hgg.), 1987, vol. II, 595-603.

Bosworth, A. B.

(1980): A Historical Commentary on Arrian's History of Alexander. vol. I, Oxford. 
(1995): A Historical Commentary on Arrian's History of Alexander. vol. II, Oxford. (1996): Alejandro Magno, Cambridge.

(1996a): Alexander and the East: the Tragedy of Triumph, Oxford.

(2003): "Plus ça change... Ancient Historians and their Sources", ClAnt 22, 167-198 (http:// dx.doi.org/10.1525/ca.2003.22.2.167).

(2004): "Mountain and Molehill? Cornelius Tacitus and Quintus Curtius", CQ 54, 551-567 (http://dx.doi.org/10.1093/cq/54.2.551).

Bouvier, D. (2004): “Quand Alexandre et Moïse voient la mer s'ouvrir devant eux ou quand Voltaire lit Flavius Josèphe. Remarques sur la référence à un miracle juif dans l'historiographie antique", [en] O. Bianchi - O. Thévenaz (éd.), P. Mudry (dir.), 'Mirabilia'. Conceptions et représentations de l'extraordinaire dans le monde antique, (= ECHO, Collection de l'Institut d'archéologie et des sciences de l'Antiquité de l'Université de Lausanne 4) Bern-Berlin-Bruxelles, etc., 171-190.

BRACCESI, L.

(1991): Alessandro e la Germania, Roma.

(2006): L'Alessandro occidentale. Il Macedone e Roma, Roma.

Brunt, P. A. (1978): “Laus imperii”, [en] P. Garnsey - C. R. Whittaker (eds.), Imperialism in the Ancient World, Cambrigde, 159-191.

Burnand, Y. - Le Bohec, J.-Y. - Martin, J.-P. (ÉD.), (1998): Claude de Lyon, Empereur Romain, Paris.

Cizek, E. (1995): Histoire et historiens à Rome dans l'Antiquité, Lyon.

Coppola, A. (1999): "Fra Alessandro e gli Eneadi, da Tiberio a Traiano", Athenaeum 87, 447456.

CORDE, X. (2005): “«Scythes justes» et «Scythes féroces»: deux traditions rélatives aux Scythes dans la Géographie de Strabon", DHA 31, 79-91.

Cornell, T. (1993): “The End of Roman Imperial Expansion", [en] J. Rich - G. Shipley (eds.), War and Society in the Roman World, London, 139-170.

Croisille, J. M. (ÉD.), (1990): Alejandro Magno, modelo de los Emperadores Romanos. Actes du IVe colloque de la SIEN (=Collection Latomus 209), Bruxelles.

Dauge, Y. A. (1981): Le Barbare. Recherches sur la conception romaine de la barbarie et de la civilisation (=Collection Latomus 176), Bruxelles.

Devillers, O.

(2014): "Fonction des discours dans les livres 6 et 7 des Histoires de Quinte-Curce", Exercices de rhétorique 3, 2-13.

(2014a): "Analogies et contrastes dans les Historiae Alexandri de Quinte-Curce. Alexandre chez les Scythes et les Sudraques”, [en] Mahé-Simon - Trinquier (éd.), 2014, 119-124.

Desnier, J.-L. (1995): De Cyrus le Grand à Julien l'Apostat, “Le passage du fleuve”. Essai sur la legitimité du souverain, Paris.

Dion, J. (1998): “Les publications littéraires sous le principat de Claude, 41-54”, [en] Burnand - Le Bohec - Martin (éd.), 1998, 69-79.

Dupont, F. (1995): “«En Germanie, cet-à-dire, à nulle part». Rhétorique de l'alterité et rhétorique de l'identité: l'aporie descriptive d'un territoire barbare dans la Germanie Tacite", [en] Rousselle (éd.), 1995, 189-219. 
ERskine, A. (2002): "Life after Death: Alexandria and the Body of Alexander", $G \& R 49,163-$ 179 (http://dx.doi.org/10.1093/gr/49.2.163).

D’Erce, F. (1966): “La tour de Caligula à Boulogne-sur-Mer”, $R A$ 1, 89-96.

FrÉZouls, E. (1995): "Les relations romano-parthes avant l'Époque flavienne”, [en] E. Frézouls - A. Jacquemin (éd.), Les relations internationales (=Travaux du Centre de Recherche sur le Proche-Orient et la Grèce Antique 13), Paris, 479-498.

Fugmann, J. (1995): “Zum Problem der Datierung der 'Historiae Alexandri Magni' des Curtius Rufus", Hermes 123, 233-243.

Gale, M. (2007): Lucretius, Oxford.

Geus, K. (2003): "Space and Geography", [en] A. Erskine (ed.), A Companion to the Hellenistic World, Oxford, 232-245.

Gissel, J. A. P. (2001): "Germanicus as an Alexander Figure”, C\&M 52, 277-302.

Goukowsky, P. (1981): Essai sur les origines du mythe d'Alexandre (336-270 av. J.-C.). Tome II, Alexandre et Dionysos, Nancy.

Hamilton, J. R. (1971): "Alexander and the Aral", $C Q$ 21, 106-111 (http://dx.doi.org/10.1017/ S0009838800028834).

(1988): “The Date of Quintus Curtius Rufus", Historia 37, 445-456.

Hammond, N. G. L.

(1983): Three Historians of Alexander the Great. The So-called Vulgate Authors, Diodorus, Justin and Curtius, Cambridge.

(1992): Alejandro Magno, rey, general y estadista, Madrid.

Hannestad, N. (1997): "Imitatio Alexandri in Roman Art", [en] J. Carlsen - B. Due - O. S. Due - B. Poulsen (eds.), Alexander the Great. Reality and Myth (=Analecta Romana Instituti Danici, Supplementum 20), Roma, 61-69.

Hartog, F. (1980): Le miroir d'Hérodote. Essai sur la représentation de l'autre, Paris.

Heckel, W. (2006): Who's who in the Age of Alexander the Great, Oxford.

Helmreich, H. (1927): Die Reden bei Curtius (= Rhetorische Studien 14), Paderborn.

Heuss, A. (1954): “Alexander der Grosse un die politische Ideologie des Altertums”, $A \& A 4$, 65-104.

HIND, J. (2003): "Caligula and the Spoils of Ocean: a Rush for Riches in the Far NorthWest?", Britannia 24, 272-274 (http://dx.doi.org/10.2307/3558547).

Hurley, D. (1993): An Historical and Historiographical Commentary on Suetonius'Life of C. Caligula (=American Classical Studies 32), Atlanta.

Ivantchiк, A. I. (1995): "Eine griechische Pseudo-Historie. Der Pharao Sesostris und der skytho-ägyptische Krieg”, Historia 48, 395-441.

Jerphagnon, L. (1981): Vivre et philosopher sous les Césars, Toulouse.

Kienast, D. (1969): “Augustus und Alexander”, Gymnasium 76, 430-456.

Kleijwegt, M. (1994): “Caligulas' Triumph at Baiae”, Mnemosyne 47, 652-671 (http://dx.doi. org/10.1163/156852594X00537).

Kolendo, J.

(1991): “Les «Déserts» dans les Pays Barbares. Représentations et réalités”, DHA 17, 35 60. 
(1998): “Claude et l'annexion de la Thrace", [en] Burnand - Le Bohec - Martin (éd.), 1998, 321-332.

Kraus, C. S. - Woodman, A. J. (1997): Latin Historians (=Greece \& Rome Supplement 27), Cambridge.

Kyriakou, P. (2006): A Commentary on Euripides'Iphigenia in Tauris, Berlin.

LASsAndro, D. (1984): "La figura di Alessandro Magno nell'opera di Seneca", [en] M. Sordi (ed.), Alessandro Magno tra Storia e Mito, Milano.

LeCoQ, P. (1986): “Artaphrenēs”, Encyclopaedia Iranica 2/6, London-Boston, 651-652.

LENS Tuero, J.

(1994): "La réplica de los árabes nabateos a Demetrio Poliorcetes", [en] Lens Tuero (ed.), 1994, 117-126.

(1994a): "La respuesta del rey escita Ateas a Filipo de Macedonia", [en] Lens Tuero (ed.), 1994, 195-200.

(1994b): “El Encuentro entre Dromijaites y Lisímaco", [en] Lens Tuero (ed.), 1994, 201-208.

Lens Tuero, J. (ED.), (1994): Estudios sobre Diodoro de Sicilia (=Biblioteca de Estudios Clásicos 1), Granada.

Lerouge-Cohen, C. (2014): “Alexandre, Rome et les Parthes dans les Histoires d'Alexandre de Quinte-Curce”, [en] Mahé-Simon - Trinquier (éd.) 2014, 199-210.

LeVI, M. A. (1977): Introduzione ad Alessandro Magno, Milano.

LÉvy, E. (1981): "Les origines du mirage scythe", Ktema 6, 57-68.

LEvick, B. (1990): Claudius, London.

Lovejoy, A. O. - BoAs, G. (1935): Primitivism and Related Ideas in Antiquity, BaltimoreLondon.

Lund, A. A. (1987): "Lexikalische und kritische Bemerkungen zu Tacitus und Curtius Rufus", Gymnasium 94, 50-56.

MacL. Currie, H. (1990): “Quintus Curtius Rufus - The Historian as Novelist?”, [en] H. Hofmann (ed.), Gröningen Colloquium on the Novel, Gröningen, t. III, 63-77.

Mahé-Simon, M. - Trinquier, J. (2014): “Avant-propos”, [en] Mahé-Simon - Trinquier (éd.), 2014, 7-27.

Mahé-Simon, M. - Trinquier, J. (ÉD.), (2014): L'Histoire d'Alexandre selon Quinte-Curce, Paris.

Malloch, S. V. J.

(2001): "Gaius' Bridge at Baiae and Alexander-Imitatio", CQ 51, 206-217 (http://dx.doi. org/10.1093/cq/51.1.206).

(2001a): "Gaius on the Channel Coast", CQ 51, 551-556 (http://dx.doi.org/10.1093/ cq/51.2.551).

Marco Simón, F. (2000): “Éschatoi Andrôn. La idealización de celtas e hiperbóreos en las fuentes griegas", DHA 26, 121-147.

Martin, R. P. (1996): "The Scythian Accent: Anacharsis and the Cynics", [en] R. Bracht Branham - M. O. Goulet-Cazé (eds.), The Cynics. The Cynic Movement in Antiquity and its Legacy (=Hellenistic Culture and Society 23), Berkeley, 136-155.

Martin, V. (1959): “Un recueil de diatribes cyniques: Pap. Genev. inv. 271”, MH 16, 77-115.

Mazzarino, S. (1966): Il Pensiero storico classico, Bari, vol. II. 
McKechnie, P. (1999): "Manipulation of Themes in Quintus Curtius Rufus' Book 10", Historia 48, 44-60.

Melmoux, J. (1990): “L'Empereur Claude et la Finium Imperii Propagatio: l'exemple Breton”, [en] Croisille (éd.), 1990, 163-182.

Montero Herrero, S.

(1990): "La religiosidad de Alejandro en la historiografía latina: el testimonio de Quinto Curcio", [en] Croisille (éd.), 1990, 339-350.

(2012): El Emperador y los ríos. Religión, ingeniería y política en el Imperio Romano (=UNED. Arte y Humanidades 16), Madrid.

Morgan, K. A. (2015): Pindar and the Construction of Syracusan Monarchy in the Fifth Century B.C., Oxford.

Muckensturm-Poulle, C. (2013): "L'agôn d'Alexandre et de Coenos au bord de l'Hyphase selon Arrien et Quinte-Curce", [en] D. Côté - P. Fleury (éd.), Discours politique et histoire dans l'Antiquité (=DHA Extra 8), Besançon, 259-275.

NDiaye, E. (2009): “Alexandre, Rome et les barbares d'Orient: rhétorique et politique dans l'Histoire d'Alexandre de Quinte-Curce (Livres VIII-X)", Vita Latina 181, 45-56 (http:// dx.doi.org/10.2143/VL.181.0.2046929).

Osgood, J. (2011): Claudius Caesar: Image and Power in the Early Roman Empire, Cambridge.

Pearson, L. (1960): The Lost Histories of Alexander the Great (=American Philological Association, Philological Monographs 20), New York.

PicARD, G.-C. (1957): Les Trophées romains. Contribution à l'histoire de la Réligion et de l'Art triomphal de Rome (=Bibliothèque des Écoles françaises d'Athènes et de Rome 187), Paris.

PlÁCIDO, D. (1990): “Alejandro y los emperadores romanos en la Historiografía griega”, [en] Croisille (éd.), 1990, 58-75.

Polignac, F. DE (1996): “Cosmocrator: l'Islam et la légende antique du souverain universel”, [en] M. Brigdes - J. Ch. Bürgel (eds.), The Problematics of Power. Eastern and Western Representations of Alexander the Great (=Schweizer Asiatische Studien, Monographien 22), Bern, 149-164.

Prandi, L. (1990): “Gli oracoli sulla spedizione asiatica di Alessandro", Chiron 20, 351-369.

Prontera, F. (1988): "Sobre la delineación de Asia en época Helenística”, [en] A. Pérez Jiménez - G. Cruz Andreotti (eds.), Los límites de la Tierra: el espacio geográfico en las culturas mediterráneas (=Ediciones Clásicas, Mediterránea 3), Madrid, 77-105.

Ramage. E. S. (2001): “The Bellum Iustum in Caesar's De Bello Gallico”, Athenaeum 89, 144-170.

RAPIN, C. (2014): "Du Caucase au Tanaïs: les sources de Quinte-Curce à propos de la route d'Alexandre le Grand en 330-329 av. J.-C.", [en] Mahé-Simon - Trinquier (éd.), 2014, 141-186.

Richard, F. (1998): "Les images du Triomphe de Claude sur la Bretagne", [en] Burnand - Le Bohec - Martin (éd.), 1998, 355-371.

Rickly, D. (1975): “Le franchissement en force du «Tanaïs» par Alexandre le Grand, QuinteCurce VII, 9", Mélanges Esther Bréget, Génève, 29-36.

Rogers, B. B. (1927): Aristophanes, vol. II, Loeb Classical Library, 221, London-New York. 
Romm, S. (1992): The Edges of the Earth in Ancient Thought: Geography, Exploration and Fiction, Princeton.

Rousselle, A. (ÉD.), (1995): Frontières terrestres, frontières célestes dans l'Antiquité (=Collection Études 20), Paris.

SÁnchez León, M. L. (1990): "Poder político y geografía a comienzos del principado: la herencia de Alejandro", [en] Croisille (éd.), 1990, 76-99.

SidARI, D. (1982): Problema partico e imitatio Alexandri nella dinastia Giulio-Claudia (=Memorie dell'Istituto Veneto di Scienze, Lettere ed Arti 38), Venezia.

Sordi, M. (1990): “Alessandro e Roma nella concezione storiografica di Orosio", [en] Croisille (éd.), 1990, 388-395.

Spencer, D. (2002): The Roman Alexander. Reading a Cultural Myth, Exeter.

Steele, R. B. (1915): "Quintus Curtius Rufus", AJPh 36, $402-423$ (http://dx.doi. org/10.2307/289627).

Stoneman, R. M. (1995): "Naked Philosophers: the Brahmans in the Alexander Historians and the Alexander Romance", JHS 115, 99-114 (http://dx.doi.org/10.2307/631646).

Szalc, A. (2011): “Alexander's dialogue with Indian philosophers: riddle in Indian and Greek tradition", Eos 98, 7-25.

TANDOI, V. (1964): “Albinovano Pedone e la retorica giulio-claudia delle conquiste I", SIFC 36, 129-168.

ThÉBERT, Y. (1995): “Nature des frontières dans l'Empire Romain: le cas Germain”, [en] Rousselle (éd.), 1995, 221-235.

Thomasson, B. E. (1998): "Provinces et gouverneurs sur Claude", [en] Burnand - Le Bohec - Martin (éd.), 1998, 229-242.

Tuplin, C. (2010): "Revisiting Dareios' Scythian Expedition", [en] J. Nieling - E. Rehm (eds.), Achaemenid Impact in the Black Sea. Communication of Powers (=Black Sea Studies 11), Aarhus, 281-312.

Wardle, D. (1994): Suetonius'Life of Caligula. A Commentary (=Collection Latomus 225), Bruxelles.

Weinstock, S. (1971): Divus Iulius, Oxford.

Wilhelm, F. (1928): Curtius und der jüngere Seneca (=Rhetorische Studien 15), Paderborn.

Will, W. - Heinrichs, G. (HGG.), (1987): Zu Alexander dem Grossen. Festschrift für Gerhard Wirth zum 60. Geburtstag am 9.12.86, Amsterdam.

Wirth, G. (1976): “Alexander und Rom”, [en] E. Badian (éd.), Alexandre le Grand, image et realité (=Entretiens sur l'Antiquité Classique XII), Vandoeuvres-Génève, 181-210.

Woods, D.

(2000): “Caligula’s Seashells", G\&R 47, 80-87 (http://dx.doi.org/10.1093/gr/47.1.80).

(2002): "Did Caligula Plan to Bridge the English Channel?", $A W 33,157-169$.

(2006/2007): "Caligula, Pompey and Alexander the Great", Eranos 104, 10-133.

(2012): "Seven notes on the reign of Caligula", [en] C. Deroux (ed.), Studies in Latin Literature and Roman History XVI (=Collection Latomus 338), Bruxelles, 437-471.

Yardley, J. C. - AtKinson, J. E. (2009): Curtius Rufus. Histories of Alexander the Great Book 10, Oxford.

Yardley, J. C. - Heckel, W. (1984): Quintus Curtius Rufus. The History of Alexander, London. 